\title{
Publisher's Note: Plethora of relativistic charged spheres: The full spectrum of Guilfoyle's static, electrically charged spherical solutions [Phys. Rev. D 95, 104040 (2017)]
}

José P. S. Lemos and Vilson T. Zanchin॰

(A) (Received 28 October 2020; published 16 November 2020)

DOI: 10.1103/PhysRevD.102.109901

This paper was published online on 30 May 2017 with an error in the title. The title should read "Plethora of relativistic charged spheres: The full spectrum of Guilfoyle's static, electrically charged spherical solutions." The paper has been corrected as of 29 October 2020. The title is incorrect in the printed version of the journal. 